\title{
Sniffer dogs can identify lung cancer patients from breath and urine samples
}

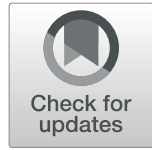

Charlotte Feil $^{1 \dagger}$, Frank Staib ${ }^{1 \dagger}$, Martin R. Berger ${ }^{2}$, Thorsten Stein ${ }^{1}$, Irene Schmidtmann ${ }^{3}$, Andreas Forster ${ }^{4}$ and Carl C. Schimanski ${ }^{*}$

\begin{abstract}
Background: Lung cancer is the most common oncological cause of death in the Western world. Early diagnosis is critical for successful treatment. However, no effective screening methods exist. A promising approach could be the use of volatile organic compounds as diagnostic biomarkers. To date there are several studies, in which dogs were trained to discriminate cancer samples from controls. In this study we evaluated the abilities of specifically trained dogs to distinguish samples derived from lung cancer patients of various tumor stages from matched healthy controls.

Methods: This single center, double-blind clinical trial was approved by the local ethics committee, project no FF20/2016. The dog was conditioned with urine and breath samples of 36 cancer patients and 150 controls; afterwards, further 246 patients were included: 41 lung cancer patients comprising all stages and 205 healthy controls. From each patient two breath and urine samples were collected and shock frozen. Only samples from new subjects were presented to the dog during study phase randomized, double-blinded. This resulted in a specific conditioned reaction pointing to the cancer sample.

Results: Using a combination of urine and breath samples, the dog correctly predicted 40 out of 41 cancer samples, corresponding to an overall detection rate of cancer samples of 97.6\% (95\% Cl [87.1, 99.9\%]). Using urine samples only the dog achieved a detection rate of $87.8 \%$ (95\% Cl [73.8, 95.9\%]). With breath samples, the dog correctly identified cancer in 32 of 41 samples, resulting in a detection rate of $78 \%$ (95\% Cl [62.4, 89.4\%]).

Conclusions: It is known from current literature that breath and urine samples carry VOCs pointing to cancer growth. We conclude that olfactory detection of lung cancer by specifically trained dogs is highly suggestive to be a simple and non-invasive tool to detect lung cancer. To translate this approach into practice further target compounds need to be identified.
\end{abstract}

Keywords: Lung cancer, Olfactory detection, Volatile organic compounds

\footnotetext{
* Correspondence: Carl.Schimanski@mail.klinikum-darmstadt.de

${ }^{+}$Charlotte Feil and Frank Staib contributed equally to this work.

2nd Department of Internal Medicine, Municipal Hospital Darmstadt, Grafenstraße 9, 64283 Darmstadt, Germany

Full list of author information is available at the end of the article
}

(c) The Author(s). 2021 Open Access This article is licensed under a Creative Commons Attribution 4.0 International License, which permits use, sharing, adaptation, distribution and reproduction in any medium or format, as long as you give appropriate credit to the original author(s) and the source, provide a link to the Creative Commons licence, and indicate if changes were made. The images or other third party material in this article are included in the article's Creative Commons licence, unless indicated otherwise in a credit line to the material. If material is not included in the article's Creative Commons licence and your intended use is not permitted by statutory regulation or exceeds the permitted use, you will need to obtain permission directly from the copyright holder. To view a copy of this licence, visit http://creativecommons.org/licenses/by/4.0/ The Creative Commons Public Domain Dedication waiver (http://creativecommons.org/publicdomain/zero/1.0/) applies to the data made available in this article, unless otherwise stated in a credit line to the data. 


\section{Background}

\section{Lung cancer: mortality, risk, and screening methods}

Lung cancer is the leading oncological cause of death in western countries and it is the second most frequent cause of death after cardiovascular diseases in Germany [1]. The WHO estimated 2.09 million newly diagnosed lung cancer patients in 2018 worldwide.

The prognosis of lung cancer is unfavorable as indicated by the death of 16.382 female and 29.692 male patients in Germany 2017 [1]. The mortality rate increases with age and reaches a maximum in the age group from 80 to 84 years [2]. An advanced age at diagnosis is considered as unfavorable prognostic factor [3]. In 2017 the median age of death was 71 years for women and 72 years for men [1].

Although early diagnosis of cancer using effective screening methods is crucial for successful treatment, no surveillance tools exist. In contrast to other cancers, there is still no early test for lung cancer [2]. Some tumor markers are available; however, due to limited sensitivity and specificity the current German S3 guideline does not recommend the routine determination of tumor markers for primary or recurrent lung cancer diagnostics, nor their use as screening tool [2].

Even if effectiveness has now been proven by some studies [3, 4], the current German S3 guideline does not yet recommend general low-dose computed tomography for screening in risk patients. Reasons among other include risks such as false positive findings with corresponding unnecessary follow-up interventions and radiation exposure.

Considering the frequency of deaths from lung cancer in Germany and worldwide the development of a screening tool for early detection of lung cancer would be of high importance, since early detection measures could lead to earlier diagnosis and thus result in better outcome.

\section{Sniffer dogs for cancer detection identifying volatile organic compounds (VOCs)}

Dogs have a highly sensitive olfactory system, which is already used in several ways. The olfactory organ of the dog is similar to the human olfactory organ but has some peculiarities that explain the better olfactory perception. Dogs can differentiate 10.000-100.000 times better between different smells compared to humans and are able to identify volatile organic compounds (VOCs) starting from a concentration of 1:1 trillion VOCs [5]. Labrador, Retriever and the German Shepherd seem to be best suited for olfactory detection [6].

Recently, much attention has been given to the use of odors emitted in the form of VOCs as diagnostic biomarkers. In several studies, sniffer dogs were trained to discriminate cancer samples (breath, urine, cancer tissue) in different media. Across published studies, a sensitivity of $78 \%$ and a specificity of $71.5 \%$ related to olfactory detection of lung cancer samples by sniffer dogs was reported [7-11]. Those study results are encouraging but published studies differ in terms of the experimental setup, kind of odor samples, sample collection methods, dogs' characteristics and dog training methods as well as in results presented in terms of detection sensitivity and specificity $[12,13]$. In addition, it could not be clarified so far what chemical compounds dogs respond to and the quantity of these compounds. As several studies show that dogs are able to detect lung cancer either in urine or in breath yielding different results, it was an important part of this study to test if the combination of both procedures could lead to a higher detection rate. Therefore, it was the aim of this study to evaluate the capability of a classically conditioned domestic dog to accurately distinguish samples of lung cancer patients of all tumor stages in urine and breath from healthy controls without preselecting included patients with regard to smoking behavior or other lifestyle habits. By combination of urine and breath samples.

\section{Methods \\ Study design}

This was a prospective, double-blind, clinical trial including 432 patients, that were recruited at the municipal hospital Darmstadt (Klinikum Darmstadt GmbH) in cooperation with a pulmonologist's office. The trial was approved by the ethics committee of the chamber of physicians Hessen, Germany (project no FF20/2016) and was conducted from May 2016 to August 2017. The study included a conditioning phase and a study phase. Patients were separated into 2 groups: Cancer patients were defined as patients with histologically proven lung cancer, and controls were defined as participants with no detectable tumor and a physiological heart and lung auscultation.

In the conditioning phase, a Labrador dog was trained with samples of 36 cancer patients and 150 control patients, in total 186 patients; afterwards, further 246 patients in the age range 45 to 80 years were included into the study phase: 41 patients presented with a biopsy confirmed lung cancer enclosing all different stages. In addition, 205 healthy individuals with no cancer history participated and served as control samples (Table 1). From each patient two breath samples and two urine samples (total samples $=864$ samples each) were collected (see Fig. 1 and Fig. 2) and were shock frozen immediately at $-80^{\circ} \mathrm{C}$.

Sample preparation and collection was highly standardized: Urine samples were self-collected from a specimen cup filled with spontaneous urine by the patient. There was no further differentiation concerning the timing of the urine sample, e.g. early morning or midstream 
Table 1 Characteristics of both patient collectives - conditioning phase and study phase

\begin{tabular}{|c|c|c|c|c|c|}
\hline \multirow{2}{*}{$\overline{\text { Collective }}$} & & \multicolumn{2}{|c|}{ Conditioning Phase $(n=186)$} & \multicolumn{2}{|c|}{ Study Phase $(n=246)$} \\
\hline & & Cancer & Control & Cancer & Control \\
\hline \multicolumn{2}{|c|}{ Total number of patients $(n)$} & 36 & 150 & 41 & 205 \\
\hline \multirow{2}{*}{\multicolumn{2}{|c|}{ Gender (female/male) }} & $17 / 19 \triangleq$ & $74 / 76 \triangleq$ & $12 / 29 \triangleq$ & $85 / 120 \triangleq$ \\
\hline & & $47.2 \% / 52.7 \%$ & $49.3 \% / 50.6 \%$ & $29.3 \% / 70.7 \%$ & $41.5 \% / 58.5 \%$ \\
\hline \multicolumn{2}{|c|}{ Age in years (mean) } & 66.72 & 62.91 & 65,63 & 61.71 \\
\hline \multirow{2}{*}{\multicolumn{2}{|c|}{ Smoker / Non-smoker }} & $17 / 19 \triangleq$ & $38 / 112 \triangleq$ & $22 / 19 \triangleq$ & $59 / 146 \triangleq$ \\
\hline & & $47.2 \% / 52.7 \%$ & $25.3 \% / 74.7 \%$ & $53.7 \% / 46.3 \%$ & $28.8 \% / 71.2 \%$ \\
\hline \multirow{2}{*}{\multicolumn{2}{|c|}{ Renal infection (yes / no) }} & $1 / 35 \triangleq$ & $7 / 143 \triangleq$ & $1 / 40 \triangleq$ & $4 / 201 \triangleq$ \\
\hline & & $2.8 \% / 97.2 \%$ & $4.7 \% / 95.3 \%$ & $2.4 \% / 97.6 \%$ & $2.0 \% / 98.0 \%$ \\
\hline \multirow{2}{*}{\multicolumn{2}{|c|}{ Regular use of alcohol (yes / no) }} & $6 / 30 \triangleq$ & $15 / 135 \triangleq$ & $6 / 35 \triangleq$ & $28 / 177 \triangleq$ \\
\hline & & $16.7 \% / 83.3 \%$ & $10.0 \% / 90.0 \%$ & $14.6 \% / 85.4 \%$ & $13.7 \% / 86.3 \%$ \\
\hline \multirow[t]{4}{*}{ Tumor entities } & SCLC & $8 \triangleq 22.2 \%$ & & $10 \triangleq 24.4 \%$ & \\
\hline & Squamous epithelial cell cancer & $11 \triangleq 30.6 \%$ & & $12 \triangleq 29.3 \%$ & \\
\hline & Adenocarcinoma & $17 \triangleq 47.2 \%$ & & $18 \triangleq 43.9 \%$ & \\
\hline & Large-cell lung cancer & 0 & & $1 \triangleq 2.4 \%$ & \\
\hline \multirow[t]{4}{*}{ UICC-Stage } & Stage I & $3 \triangleq 8.3 \%$ & & $2 \triangleq 4.9 \%$ & \\
\hline & Stage II & $6 \triangleq 16.7 \%$ & & $6 \triangleq 14.6 \%$ & \\
\hline & Stage III & $13 \triangleq 36.1 \%$ & & $11 \triangleq 26.8 \%$ & \\
\hline & Stage IV & $14 \triangleq 38.9 \%$ & & $22 \triangleq 53.7 \%$ & \\
\hline
\end{tabular}

urine in order to avoid any sample preselection. All participants were asked for signs of urinary tract infections; results of urine rapid tests were incorporated if they were already available. For breath samples all test tubes were prepared under aseptic conditions by one person

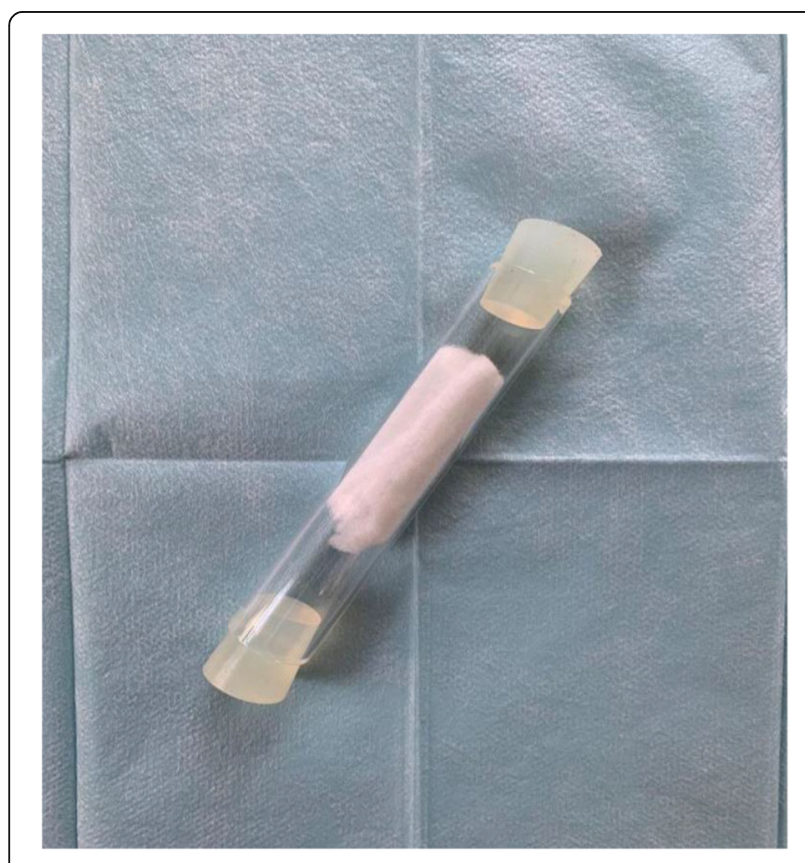

Fig. 1 Example of a test tube (breath sample) from the study team wearing aseptic gloves. Furthermore, the same person assisted each patient during sample acquisition within the study, and all samples were collected in the same room of the municipal hospital Darmstadt to avoid any contaminating smell as well as to achieve constant conditions during the sample preparation and acquisition process. Test tubes were prepared according to the process described by Ehmann et al. [9]: using 120x22mm glass tubes (Gaßner, Glastechnik $\mathrm{GmbH}$, München, Germany). They were filled with one

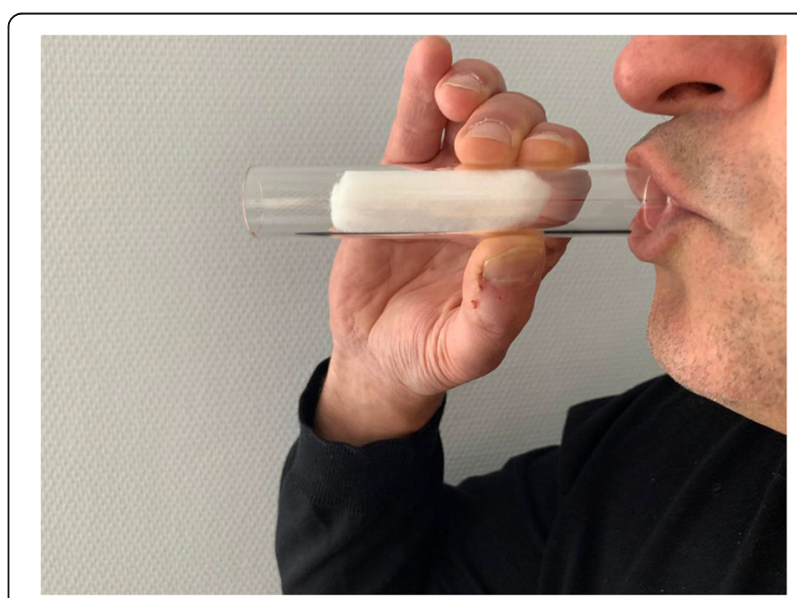

Fig. 2 Presentation of the handling of the breath tubes during delivery of the breath samples 
hydrophobic and one hydrophilic siliconized $2.5 \times 6.0 \mathrm{~cm}$ piece of polypropylene cotton wool (cotton wool by Asota Ges.m.b.H, Linz Austria; siliconization by CHT Bezema, CHT R. Breitlich GmbH, Tübingen, Germany). During preparation of test tubes and sample collection the working area was cleaned and disinfected using Cleanisept Wipes (Dr. Schumacher GmbH, Malsfeld, Germany), covered by Molinea Plus 40x60 cm tissues (Paul Hartmann AG, Heidenheim, Germany). The study team members wore aseptic gloves, surgical caps, face mask and sterile gowns.

One urine and one breath sample of each patient was preserved for another study at the German Cancer Research Center, and the other one was used in this study for presentation to the dog. In a randomized, doubleblinded manner breath and urine samples were presented separately to the dog. This resulted in a specific conditioned reaction pointing to the cancer sample. In contrast to most other studies, only samples from new subjects were presented to the dog during study phase. This was to rule out that the dog reacted to an already known odor instead of identifying the specific cancer odor.

\section{Eligibility criteria}

Participants of both groups (cancer group and control) were $45-80$ years of age and able to provide informed consent. Eligible patients for the cancer group had histologically confirmed lung cancer. Patients with completely cured tumors, other malignancies, with tumors of other than bronchial origin or with lung metastases originating from another cancer were not eligible for this study.

Patients of the control group were either hospitalized in the municipal hospital Darmstadt for other reasons, or they were hospital staff or patients from the nearby pulmonologists office in Darmstadt. Healthy volunteers were also accepted. Their samples were taken at the study's office in the municipal hospital Darmstadt. All included patients of the control group as well as all healthy volunteers had no cancer history and a nonpathological heart and lung auscultation.

\section{Study evaluations}

Cancer patients underwent a lung function test less than 5 days prior to bronchoscopy using a bodyplethysmograph "MasterScreen Body", Carefusion Germany GmbH. Only forced expiratory volume (FEV1) was collected for the study with a FEV1 $>60 \%$ of the target value corresponding to a minor obstruction, a FEV1 $=40-60 \%$ of the target value corresponding to a medium obstruction and a FEV1 $<40 \%$ corresponding to a major obstruction. A FEV1 of $>80 \%$ was defined as normal. All bronchoscopies were performed using a bronchoscope of Olympus Medical Systems Corp., series EVIS EXERA III (BF-P190 or
BF-1TH190) or series EVIS EXERA II (BF-1 T180). The bronchoscopy was evaluated as abnormal if the investigators were able to display direct or indirect tumor signs. At each bronchoscopy, samples of endobronchial tumor, the bronchial mucosa, or lung tissue (transbronchial biopsy) and adjacent lymph nodes were collected, and a lavage cytology was obtained.

In addition, the local lymph node situation and, if necessary, the spread of the primary tumor was evaluated using endobronchial ultrasonography (EBUS, PENTAX Europe GmbH, Ultrasound-Videobronchoscope EV-1970UK).

All tissue samples were analyzed histopathologically at the Department of Pathology at the Municipal Hospital Darmstadt and distinction was made between a small cell lung cancer (SCLC) and a non-small cell lung cancer (NSCLC).

In all patients with confirmed lung cancer a computer tomography (CT) was done at the Department of Radiology $\mathrm{t}$ and the tumor markers carcinoembryonic antigen (CEA), cytokeratine fragment (CYFRA) 21-1 and neuronspecific enolase (NSE) were determined at the central laboratory of the Municipal Hospital Darmstadt.

\section{Sniffer dog, its conditioning process and study setup}

Samples were evaluated by a 7-year old Golden Retriever dog trained for one year by a professional dog trainer of TEAMCANIN, Filderstadt, Germany. The training of the dog during the conditioning phase as well as sample detection during the study phase took place at the dog training facilities of TEAMCANIN. Otherwise the dog was living at his home place together with its owner. This dog was already trained to discriminate different smells e.g. chamomile tea, cinnamon or coffee.

For this study, the dog was conditioned by a classical conditioning method, called the clicker method: a correct indication of a sample was initially rewarded by food along with a specific click from a clicker device. During the conditioning phase the dog was trained to accept the click sound as the only positive reinforcement sign. Duration of the conditioning phase was about one year with training once or twice a week. Week days and training hours changed randomly. During training time the dog was able to move freely, thus he was not leashed. First, only cancer samples were presented to the dog, afterwards more and more control samples were presented together with the cancer sample until the setup goal of the study phase was achieved (one cancer sample and five control samples).

Samples were presented in an aluminum tray and each sample was covered with a funnel that was discarded after each presentation to ensure that the dog's nose was not affected by the sample's smell. The dog was standing during sample presentation, sniffing the samples with its 
nose and indicating a cancer sample by pausing more than five seconds with its nose in the funnel of a sample.

Samples involved in the conditioning phase were kept in a standard freezer at $-20{ }^{\circ} \mathrm{C}$ until they were presented to the dog.

Urine and breath samples were always presented separately.

The position of the cancer sample was chosen by dice and the sample was positioned in the aluminum tray as indicated by the dice number. This process was blinded to the dog and its trainer. Dog and owner entered the room with the samples to be studied only after the assistant had left. When the dog identified a sample as cancer sample as it was taught, the trainer reported the number of the sample to his assistant waiting in the room next to the study room. The assistant replied whether the dog's identification was correct, and the dog was then rewarded or not. The dog was rather quick sniffing the samples and indicating a cancer sample one round of sample detection lasted about $10 \mathrm{~s}$.

The study phase took place during May 2017 and August 2017.

\section{Study phase}

Each urine and breath sample was evaluated only once and was successfully presented to the dog in a randomized, double-blinded manner. In total 41 cancer samples and 205 control samples were evaluated per sample type. Only samples that had not been used during the conditioning phase were evaluated during the study phase to make sure that the dog did not recognize the smell of an already known sample.

\section{Statistics}

Results were recorded in an Excel 2010 table and stored at the 2nd Medical Department II of the Municipal Hospital Darmstadt.

For the main outcome the detection rate was computed as the proportion of correctly identified cancer samples, this was compared to $78 \%$ using one-sided exact binomial tests, since $78 \%$ was the mean values for sensitivity to the literature $[9-11,14,15]$ and the proportion of correctly identified cancer samples is approximately equal to the sensitivity. In addition, 95\% Pearson Clopper confidence intervals were provided.

To assess statistical significance in subgroup comparisons, Pearson Chi-square test and the Fisher exact test were used. Results were considered statistically significant if the $p$-value was $\leq 0.05$.

A correction for multiple testing was not done as the intent of the analysis was exploratory. Missing values were excluded from the statistical analysis.

While the design of the study did not allow to compute sensitivity and specificity of the sniffer dog, a formula was derived which related underlying sensitivity and specificity to the probability of correctly identifying the cancer sample among six samples presented to the dog. Thus, pairs of values for sensitivity and specificity could be obtained that were compatible with the observed detection rates. Further, lower bounds for sensitivity and specificity, given the observed detection rates, could be computed.

With $\mathrm{s}=$ sensitivity, $\mathrm{r}=1-$ specificity and ${ }^{+}$the probability of correctly detecting the cancer sample, it holds that $s=r \frac{6 \pi^{+}-(1-r)^{5}}{1-(1-r)^{5}}$. The derivation of the formula and details of the calculations are provided as supplementary material. Possible pairs of values of values for sensitivity and specificity are displayed graphically.

\section{Results}

\section{Patient collectives}

Conditioning phase: Urine and breath samples of 186 patients were collected from which 36 patients were assigned to the cancer group and 150 to the control group. The mean age was 63.7 years in the control group and 66.7 years in the cancer group. Slightly more men than women participated $(50.6 \%$ vs 49.3 and $52.7 \%$ vs $47.2 \%$, respectively) and the gender distribution was almost equal in both groups.

Study phase: Urine and breath samples of 246 patients were collected who had not participated before in the conditioning phase. Out of those, 41 patients were included in the cancer group and 205 patients in the control group. The mean age was 62.4 years (control) and 65.6 years (cancer group), respectively. The proportion of males and females in the cancer and control group did not differ significantly $(p=0.145)$ : the cancer group consisted of 29 males (70.7\%) and 12 females (29.3\%), and the control group of 120 males (58.5\%) and 85 females (41.5\%). Signs for urinary tract infections had been found in about $2 \%$ in the cancer and control group. Characteristics of both groups are shown in Table 1.

The tumor entities of the study phase were distributed as shown in Fig. 3.

Most of the lung cancer diseases were diagnosed as UICC stage 4 (53.7\%), followed by UICC stage 3 (26.8\%). Only $4.9 \%$ of the patients were in UICC stage 1 and $14.6 \%$ were in UICC stage 2 . The distribution of UICC stages was similar in both, the study phase and the conditioning phase.

\section{Bronchoscopy, CT, lung function tests, tumor marker}

Bronchoscopy revealed endobronchial pathological findings in 23 of 41 cancer patients resulting in a sensitivity of $56.1 \%$. The lowest sensitivity was achieved in the diagnosis of adenocarcinomas (33.3\%). It was not possible to 


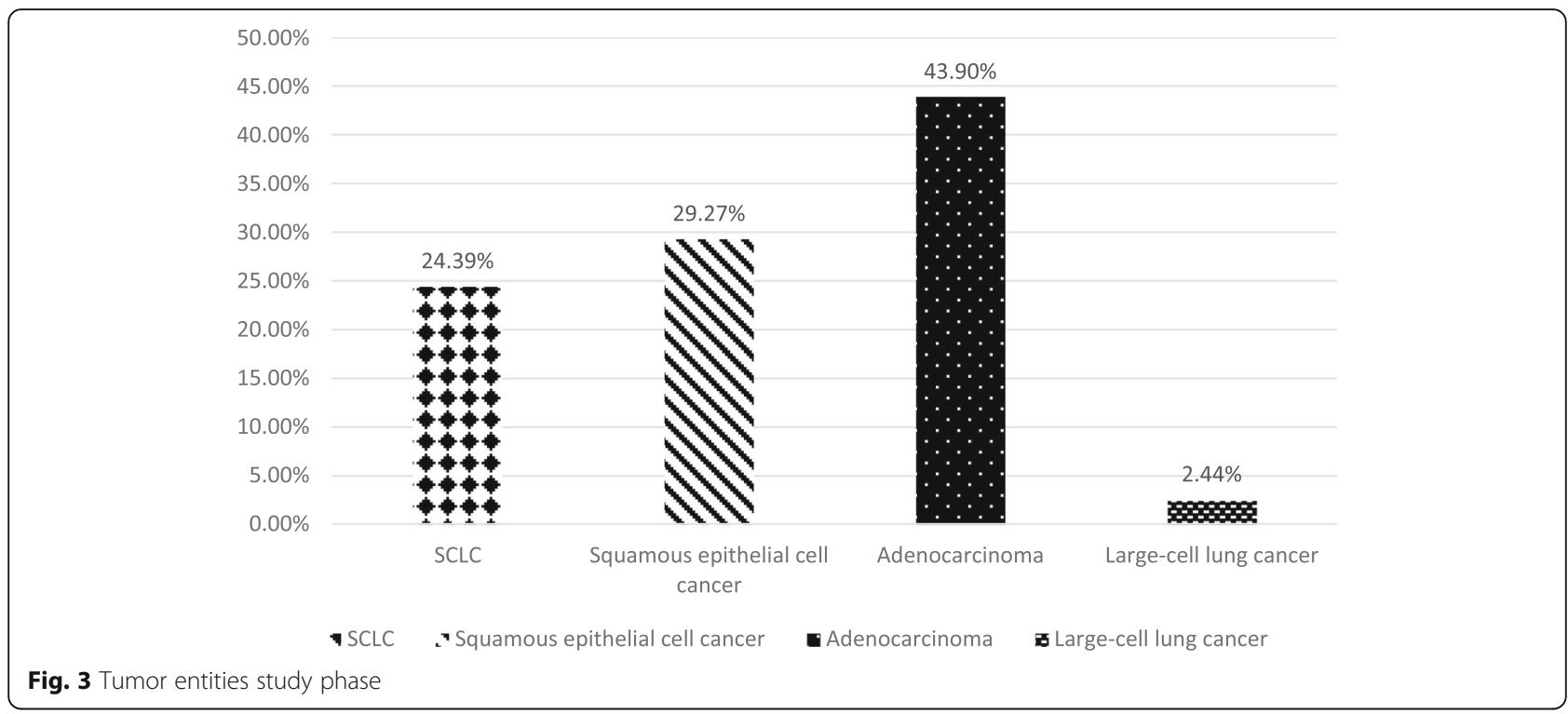

determine the specificity of the method since no bronchoscopy was done in patients of the control group.

By CT examination $100 \%$ of carcinomas were diagnosed correctly; again, the specificity of this method could not be determined, as control group patients did not receive CT diagnostics.

Data of the lung function test (FEV1) were available in 35 patients. Mean FEV1 was $62.8 \% \pm 23.0 \%$ corresponding to a mild obstruction. In 9 out of 35 patients, there was no pathological finding.

Results of tumor markers (CYFRA 21-1, CEA, NSE) were available in 30 patients. For 29 out of these 30 patients at least one tumor marker was positive. NSE was the tumor marker showing the highest sensitivity with 93.1\%; specificity of the method was not determined. In Fig. 4 comparison of the sensitivity of the diagnostic procedures compared to the detection rates using breath samples (approximately equal to sensitivity) is shown.

\section{Results of breath tests - detection rates}

The dog correctly predicted cancer in 32 of 41 breath samples, corresponding to a detection rate of $78 \%(95 \%$ CI [62.4, 89.4\%]). This did not differ significantly from the reference value of $78 \%(p=0.5851)$. Results are depicted in Table 2.

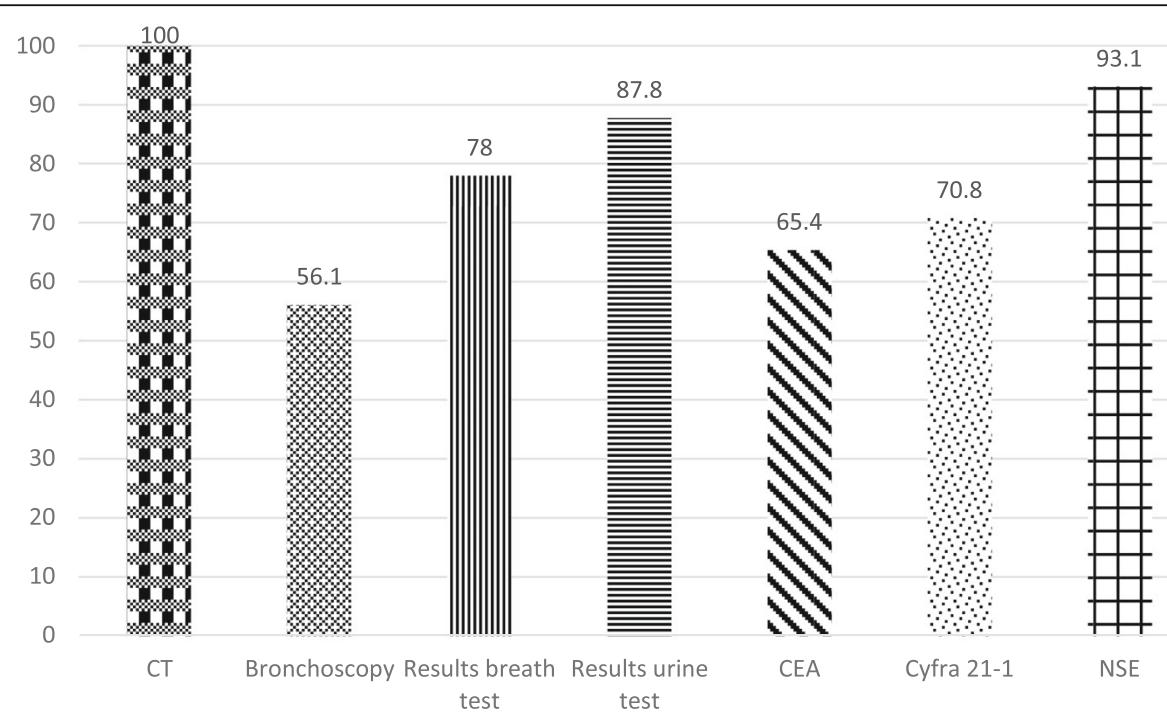

Fig. 4 Comparison of the sensitivity of the diagnostic procedures in relation to detection rates in lung cancer using breath tests 
Table 2 Evaluation of breath samples: Detection rates

\begin{tabular}{llll}
\hline Results breath samples & \multicolumn{3}{l}{} \\
\hline & \multicolumn{2}{l}{ Sample chosen } & Total \\
\cline { 2 - 3 } & cancer & control & \\
\hline Number of sample groups & 32 & 9 & 41 \\
Percentage & $78.0 \%$ & $22.0 \%$ & $100.0 \%$ \\
\hline
\end{tabular}

Looking at tumor entities the highest detection rate (90.0\%) was found for SCLC. Large-cell lung cancers were not evaluated in detail since there was only one patient present with this diagnosis. Detection rate of adenocarcinomas was 72.2 and $74.2 \%$ for the total group of NSCLC. However, differences between histological subtypes are not statistically significant $(p=0.710)$. Results are summarized in Table 3.

Considering the Union for International Cancer Control (UICC) stages the detection rate of the breath test was not evaluable at UICC stage 1 due to the low case number. Detection rate improved with higher UICC stages and was $66.7 \%$ at UICC stage $2,72.7 \%$ at UICC stage 3 , and $81.8 \%$ at UICC stage 4 , respectively. The differences in detection rates between stages were not statistically significant $(p=0.793)$.

The detection rate in cancer patients with a normal FEV1 was $88.9 \%$. In patients with severe obstruction, it was $83.3 \%$. The differences in detection rates of the breath test by FEV1 were not statistically significant with $p=0.646$.

Correlating test results with bronchoscopy findings, there was no statistically significant difference in the detection rates of the breath test $(p=0.970)$.

The lower bound for sensitivity (at specificity $=100 \%$ ) was derived as $73.7 \%$. Similarly, specificity was at least $90 \%$ (at sensitivity $=99.9 \%$ ). For all possible pairs of values for sensitivity and specificity see Fig. 5 .

\section{Test in urine - detection rate}

The dog correctly predicted cancer in 36 of 41 samples, resulting in a detection rate of $87.8 \%$ (95\% CI [73.8\%, 95.9. The detection rate did not differ significantly from the reference value of $78 \%(p=0.0865)$. Results are depicted in Table 4.

Evaluating the detection rate of the test in relation to tumor entities the highest detection rate (91.7\%) was found for squamous epithelial cell cancer. Again, due to the low case number, large-cell lung cancers were not taken into consideration. Detection rate for the detection of SCLC was $90.0 \%$ and it was $83.3 \%$ for adenocarcinomas. However, differences between histological subtypes are not statistically significant $(p=0.865)$. Results are summarized in Table 5.

Looking at UICC stages the highest detection rate in detection of cancer samples could be demonstrated for samples of patients with UICC stage 3 (100.0\%) followed by samples of patients with UICC stage 4 (86.4\%). However, results were not statistically significant.

Correlating test results with bronchoscopy findings, there was no statistically significant difference in the detection rate of the urine test.

The lower bound for sensitivity (at specificity $=100 \%$ ) was derived as $85.4 \%$. Similarly, specificity was at least $94.8 \%$ (at sensitivity $=99.9 \%$ ). For all possible pairs of values for sensitivity and specificity see Fig. 5.

\section{Comparison of the results of the test in breath and in urine by tumor entity and UICC stage}

Combining both techniques, the dog correctly identified 40 of 41 cancer samples, leading to an overall detection rate of $97.6 \%(95 \% \mathrm{CI}[87.1,99.9 \%])$ which was significantly higher than the reference value of $78 \%(p=$ 0.0005). SCLCs were detected with equal frequency in both breath and urine samples. Samples of adenocarcinoma as well as of squamous epithelial cell cancer were detected slightly more frequently in urine samples but

Table 3 Evaluation of breath samples: Detection rates by tumor entity

\begin{tabular}{|c|c|c|c|c|}
\hline \multirow[t]{2}{*}{ Tumor entity } & & \multicolumn{2}{|c|}{ Results breath test } & \multirow[t]{2}{*}{ Total } \\
\hline & & false & correct & \\
\hline \multirow[t]{2}{*}{$\mathrm{SCLC}$} & Number of samples & 1 & 9 & 10 \\
\hline & Percentages & $10.0 \%$ & $90.0 \%$ & $100.0 \%$ \\
\hline \multirow[t]{2}{*}{ Squamous epithelial cell cancer } & Number of samples & 3 & 9 & 12 \\
\hline & Percentages & $25.0 \%$ & $75.0 \%$ & $100.0 \%$ \\
\hline \multirow[t]{2}{*}{ Adenocarcinoma } & Number of samples & 5 & 13 & 18 \\
\hline & Percentages & $27.8 \%$ & $72.2 \%$ & $100.0 \%$ \\
\hline \multirow[t]{2}{*}{ Large-cell lung cancer } & Number of samples & 0 & 1 & 1 \\
\hline & Percentages & $0.0 \%$ & $100.0 \%$ & $100.0 \%$ \\
\hline
\end{tabular}




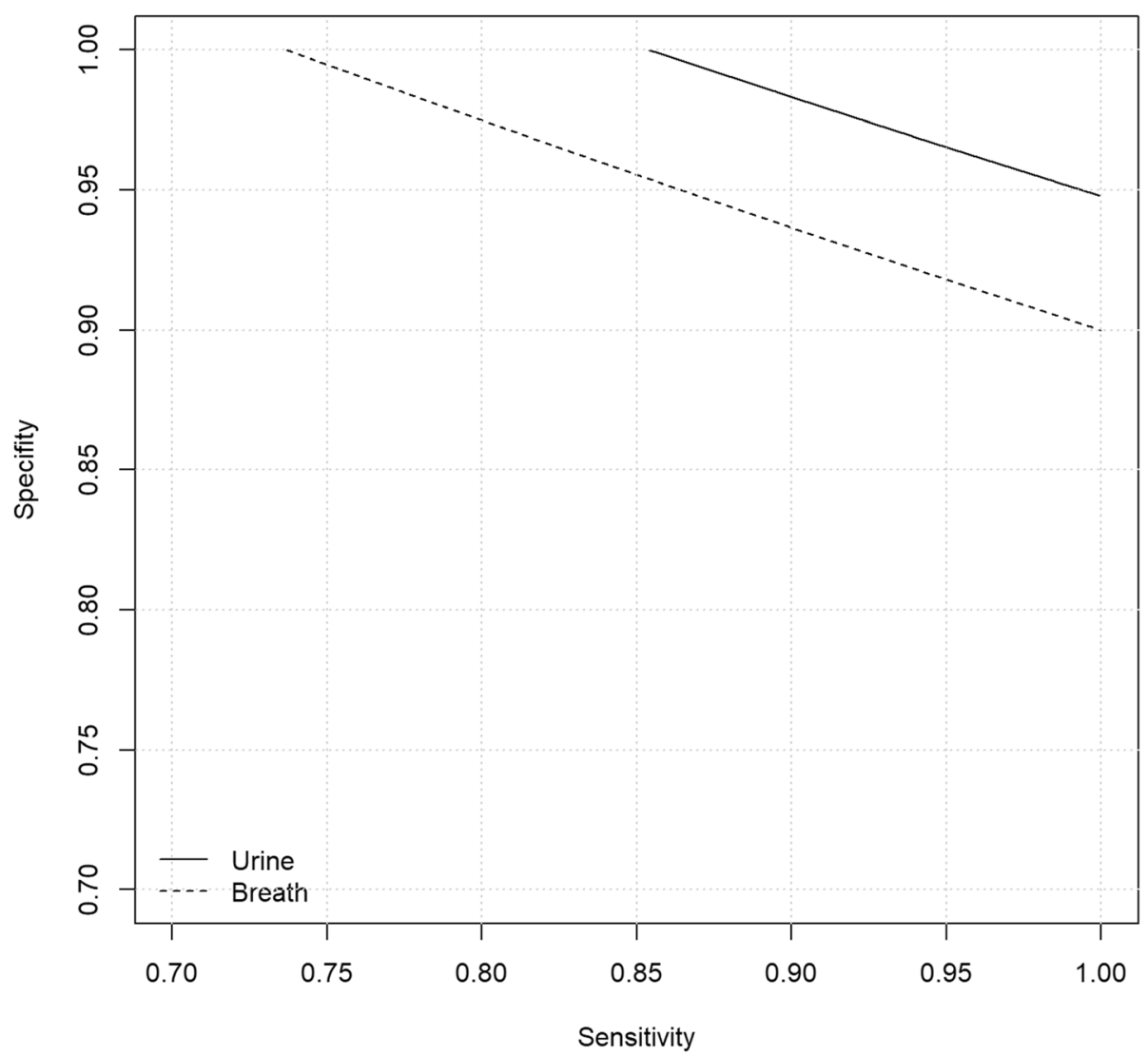

Fig. 5 Combinations of sensitivity and specificity compatible with observed detection rates

this difference was not statistically significant. UICC stage 2-4 cancer was more frequently found by the dog in urine samples than in breath samples.

\section{Discussion}

It was the purpose of this study to evaluate the capability of a classically conditioned domestic dog to accurately discriminate urine and breath samples from lung cancer patients of all tumor stages from healthy controls in a strongly standardized and controlled setting of a prospective trial.

\section{Study population}

The study population as defined by the in- and exclusion criteria reflected the population in which the development of cancer is more likely and would therefore benefit most

Table 4 Evaluation of urine samples - detection rate Results urine samples

\begin{tabular}{llll}
\hline & \multicolumn{2}{l}{ Sample chosen } & Total \\
\cline { 2 - 3 } & cancer & cancer & \\
\hline Number of sample groups & 36 & 5 & 205 \\
Percentage & $87.8 \%$ & $12.2 \%$ & $100.0 \%$ \\
\hline
\end{tabular}

from lung cancer screening. Based on the data of the German S3-guideline for lung cancer, males are more frequently affected by lung cancer than females [2], hence 149 male and 97 female patients participated in this study. According to the Robert Koch Institute, the mean age of disease onset was 66 years in males and 64 years in females in 2016 [1]. The incidence increases starting at the age of $35-39$ years and peaks at the age of $80-84$ years. The mean age of the study population was 65.6 years corresponding to the mean age of patients published by the Robert Koch Institute, however, an extension of the inclusion age up to and including 85 years would have defined the affected age group more precisely.

Some authors describe that the amount of dissolved substrates or VOCs in exhaled air depends on the lung volume [16]. Therefore, FEV1 values of cancer patients were determined. We could not confirm that the ability of dogs to correctly detect cancer samples was compromised when evaluating samples from patients with a limited FEV1. In this study, the detection rate in breath samples from patients with normal FEV1 was only slightly better than that of breath samples from patients with severe obstruction $(88.9 \%$ vs. $83.3 \%)$. However, this statement is impaired by the low number of patients with a medium or 
Table 5 Evaluation of urine samples - detection rate by tumor entity

\begin{tabular}{|c|c|c|c|c|}
\hline \multirow[t]{2}{*}{ Tumor entity } & & \multicolumn{2}{|c|}{ Results urine test } & \multirow[t]{2}{*}{ Total } \\
\hline & & false & correct & \\
\hline \multirow[t]{2}{*}{ SCLC } & Number of samples & 1 & 9 & 10 \\
\hline & Percentages & $10.0 \%$ & $90.0 \%$ & $100.0 \%$ \\
\hline \multirow[t]{2}{*}{ Squamous epithelial cell cancer } & Number of samples & 1 & 11 & 12 \\
\hline & Percentages & $8.3 \%$ & $91.7 \%$ & $100.0 \%$ \\
\hline \multirow[t]{2}{*}{ Adenocarcinoma } & Number of samples & 3 & 15 & 18 \\
\hline & Percentages & $16.7 \%$ & $83.3 \%$ & $100.0 \%$ \\
\hline \multirow[t]{2}{*}{ Large-cell lung cancer } & Number of samples & 0 & 1 & 1 \\
\hline & Percentages & $0.0 \%$ & $100.0 \%$ & $100.0 \%$ \\
\hline
\end{tabular}

SCLC: Small cell lung cancer

severe obstruction. Mass spectroscopic analyses are warranted to get further insights into the influence of a low flow perhaps resulting in a lower concentration of VOCs.

\section{Sniffer dogs can distinguish VOCs from cancer patients and set up during the study}

The race of the conditioned dog as well as factors like age, diet, motivation or health status could influence the suitability of a dog. To get more objective results it might be useful to have the same samples evaluated by several dogs, as it was already tried in other studies [9, 13, 17, 18]. Another factor influencing detection results had been demonstrated by Biehl et al., who described different outcomes depending on previous experiences of the dog, e.g. police dog vs. household dog vs. tumor sniffing dog with a better outcome for experienced dogs. However, due to the low number of dogs included in detection dog studies, it is difficult to prove an influence of the individual dog on the result statistically $[13,17]$.

Dogs have a pronounced olfactory memory. Therefore, repeatedly presented patient samples during the conditioning and/or the study phase may result in a dog recognizing an already known sample and thus being incorrectly conditioned to the detection of already presented samples rather than to the olfactory detection of cancer-specific VOCs. In order to exclude this possibility, all presented patient samples had been presented only once during the study phase. This procedure reduced bias to a minimum due to residence or food within the same patient. Control of any lifestyle factor was not intended, as the aim of this study was to develop a screening method for lung cancer that could be used everywhere and for everyone, independent from the lifestyle. While the development of an electronic nose is the ultimate goal of our study, the sniffer dog's identification of lung cancer, as presented here, was only the first step towards the electronic nose and will be followed by identification of a VOD signature within the same samples that had been analyzed here. However, we employed highly stringent criteria to avoid any bias due to smells derived from preparation of test tubes, sample preparation and acquisition. The carefully controlled preparation of our test tubes is described in the Material and Methods section; the same study group member preparing the test tubes for breath samples was also assisting patients during sample acquisition. Furthermore, all samples were taken in the same room of the municipal hospital Darmstadt. Similarly, during the engagement of the sniffer dog, actions were taken to rule out any cross contamination of samples or smells. For example, the setting for the sniffer dog was always prepared by the same person, which was different from the person accompanying the dog; funnels applied to the test tubes facilitating the dog sniffing at each sample were used only once; position of positive samples among the negative samples was randomly assigned and blinded to the person leading the dog.

In the present study, encouraging results of the olfactory detection of lung cancer from urine and breath samples of patients could be shown. Experimental studies using trained dogs to identify breath odor markers of human cancer have been analyzed and compared with the authors' own results. The mean sensitivity reported in all previously published studies dealing with the olfactory detection of lung cancer was $78 \%$, the mean specificity was $71.5 \%[9-11,14,15]$.

Our results concerning sensitivity for the urine samples with a lower bound of $85.4 \%$ were better than the reference value of $78 \%$. Breath samples alone might be inferior regarding sensitivity - but only if specificity is above $98 \%$. Specificity was substantially better than the reference value of $71.5 \%$, both for breath and urine samples. Combining breath and urine samples is likely to lead to higher sensitivity as in 40 of 41 cases the cancer sample was detected, this will come at the cost some decrease in specificity, however, it is unlikely that the combined specificity will be below $85 \%$. 
A limitation of the design is that was impossible to obtain precise estimates for sensitivity and specificity. In order to obtain not only bounds but precise values for sensitivity and specificity, some kind of replication of the sniffing experiment with the same dog would be necessary.

It could be shown that the dog was generally better in detecting lung cancer from urine and breath samples than bronchoscopy. In this study, a sensitivity of only $56.1 \%$ could be achieved by bronchoscopy vs a sensitivity of at least $84.5 \%$ in urine and of at least $73.7 \%$ in breath samples by olfactory detection. With a sensitivity of $100 \%$, CT is the diagnostic gold standard but due to the associated radiation exposure, its use should be limited to a population at risk. The use is also limited by the high rate of false positive results [19]. Here, the combined analysis of urine and breath samples yielding an identification of 40 out of 41 samples corresponding to a detections rate of $97.6 \%$ is a promising approach, which might fill this diagnostic gap.

Thus, the olfactory detection of lung cancer is a promising alternative: The collection of breath and/or urine samples is non-invasive and there is no risk for the patient. Therefore, a precise specification of a risk population is not necessary, since even with a sensitivity and specificity $<100 \%$ there would be a benefit for the patient. Due to the simplicity of the sample collection, this test would be feasible at many locations; it is less expensive than a CT examination, requiring a relevant radiation exposure and a contrast medium with their associated risks and can be repeated at any time. Another major advantage of sniffer dogs is that they could start patient screening right after their training without further delay for patients at risk, while the development of an electronic nose requires a yet to determine profound understanding of the VOCs indicating lung cancer with comparable sensitivity and specificity.

Disadvantages and limitations of this method - general aspects: Lung cancer is among the most common cancer entities, therefore a high number of sufficiently trained dogs would be required, which seems to be unrealistic. Training of dogs is very individual and time consuming and needs to be performed by a number of professional dog trainers taking race, age, previous experience, etc. into account. Furthermore, the sniffer dogs would have to maintain their high level of training over a long time period and - even in case this could be feasible - their performance still depends on their daily status and motivation as living individuals. Considering these aspects only, this seems to be hardly feasible in clinical practices of family doctors. In addition, factors that could disturb the olfactory detection by sniffer dogs needs to be identified.
There is still another point, which had already been discussed in the literature and is of importance: It could be assumed that, apart from the smell of smoke or the change in VOCs, e.g. due to a urinary tract infection, other circumstances such as drugs or hospital odor could influence the dogs' accuracy [12]. Finally, variables like room temperature, climate conditions or humidity might also affect the sensitivity and specificity of sniffer dogs during the patient screening process.

Since it is not known which VOCs or combination of VOCs indicates cancer, it is hardly possible to determine processes in the body or environmental factors, which might have an influence on the expression or detection of these VOCs.

A study on chronic obstructive pulmonary disease (COPD) patients identified drugs as possibly disturbing factors but the inflammation itself was excluded [9]. In addition, the specific scent of hospitals was identified as potentially disturbing in other studies [12, 20].

In a study by McCulloch et al. household dogs were trained to accurately distinguish breath samples of lung and breast cancer patients from those of controls. A correlation between current tobacco consumption and the sensitivity of the olfactory detection could be demonstrated [7]. In contrast, in a study of Ehmann et al. [9] lung cancer was identified with an overall sensitivity of $71 \%$ and a specificity of $93 \%$ but the detection was independent from the presence of tobacco smoke and food odors. However, if tobacco consumption should result in a poorer sensitivity and specificity of olfactory detection of lung cancer, this method would be not suitable for screening.

In conclusion, lung cancer screening by sniffer dogs seems to be affected by too many variables to become highly reliable. Therefore, the aim is to identify electronically the VOCs that indicate cancer.

\section{Application of an electronic nose in cancer detection}

Using an electronic nose for diagnosing cancer had been used for a variety of different cancers with lung cancer being the most intensively studied. Bladini $\mathrm{C}$ et al. provided an extensive overview on the variety of cancers studied as well as the current technically available systems discussing their advantages and disadvantages [21]. The authors identified 37 articles dealing with commercial and non-commercial electronic nose systems published until January 2020. These studies used various statistical approaches, e.g., principle component analysis, support vector machine and logistic regression analysis, to identify "the lung cancer breathprint" being able to distinguish between healthy controls and lung cancer in different stages. Among these Li et al. [22] used an array of 14 different sensors in combination with an in depth data pre-processing and, among others, support vector 
machine processing for classification, reaching a sensitivity of $91.6 \%$ and a specificity of $91.7 \%$. The authors concluded that these studies displayed satisfying results with different technologies, which could be used in clinical practice with desirable technological development [21].

In contrast, identification of a breathprint of lung cancer patients using currently available electronic nose systems was the approach presented in this study: Identification of VOC biomarkers by analysis of exhaled breath in lung cancer and non-cancer samples.

Although 24 VOCs have been identified to date as potential lung cancer markers, e.g. aldehydes, 2-butanone and 1-butanol [23], there is little consensus among studies dealing with identification of lung cancer-specific VOCs. In addition, only few studies link VOC levels in patients' breath with approaches that employ sniffer dogs, as we will do following this study. This approach is to serve as positive reinforcement.

For a successful olfactory detection of lung cancer it is crucial to understand which compounds are tumorspecific, in order to use such VOCs as true tumor markers. As already mentioned, previous studies comparing the dog's detection rates of synthetic samples vs cancer samples achieved varying results [24-26]. Lung cancer cells seem to produce VOCs, which differ from normal cells, or a larger amount of VOCs compared to normal cells. Sponring et al. demonstrated that certain compounds like 2-ethyl-1-hexanol and 2-methylpentane can be cancer cell derived and thus may be indicative of the presence of a tumor [27]. Another difference between normal cells and cancer cells is the increased metabolism of substances like several types of aldehydes and butyl acetate by cancer cells resulting in a lower concentration of those VOCs [27]. Currently available literature suggests that a combination of several VOCs may indicate the presence of cancer better than a single cancer-specific VOC, [12]. Buszewski et al. [25] stated, that the signature odor of cancer that dogs use for differentiation between samples may be related to specific qualitative or quantitative olfactory impressions produced by a mixture of VOCs.

The identification of cancer-specific VOCs will therefore be a challenging objective of further studies in order to develop a non-canine screening method for lung cancer that provides an identical or higher sensitivity and specificity as achieved by the canine nose. Among these studies Li W et al., outlined a research protocol for construction of a model for early prediction of lung cancer based on exhaled biomarkers using gas chromatographymass spectrometry [22]. As follow-up analysis of the study presented here, we will use the second sample of each study participant to identify potential target molecules for cancer detection by mass spectrometry similar to the outline published by Li W et al. [22].

\section{Conclusions}

It is known from current literature that breath and urine samples carry VOCs pointing to cancer growth. We conclude that olfactory detection of lung cancer by specifically trained dogs is highly suggestive to be a simple and non-invasive tool to detect lung cancer. To translate this approach into practice further target compounds need to be identified.

\section{Abbreviations \\ CEA: carcinoembryonal antigen; COPD: chronic obstructive pulmonary disease; CT: computer tomography; CYFRA: cytokeratine fragment; EBUS: endobronchial ultrasonography; EBUS-TBNA: EBUS controlled transbronchial needle aspiration; FEV: forced expiratory volume; NSCLC: non- small cell lung cancer; NSE: neuronspecific enolase; SCLC: small cell lung cancer; UICC: Union internationale centre le cancer; VOC: volatile organic compound}

\section{Acknowledgements}

We would like to thank Thomas Riemann-Seibert, Chairman of the board of the Heidrun-Seibert Stiftung, Dieburg, Germany, who proposed the idea behind the presented study to CS. We would also like to thank the Heidrun-Seibert Stiftung for financial support.

\section{Authors' contributions}

All authors made contributions to the study concept and design; CF, TS, and AF acquired study participants, samples or data; CF, FS, MRB, TS, IS, CS interpreted data; IS provided support for statistical analysis; CF wrote the manuscript; FS, MRB, TS and CS proofread and revised the manuscript. All authors read and approved the final manuscript. CF and FS contributed equally (shared first authorship).

\section{Funding}

This study was supported by grants from the Heidrun-Seibert Stiftung, Dieburg, Germany. The funding organization was not involved in the design and implementation of the study; collection, management, analysis, and interpretation of the data; preparation, review, or approval of the manuscript; or the decision to submit the manuscript for publication.

Availability of data and materials

All data generated or analyzed during this study are included in this published article.

\section{Declarations}

\section{Ethics approval and consent to participate}

The study and all experimental protocols, which also include the involved sniffer dog, were approved by the local ethics committee (at the national physician chamber Hessen in Frankfurt, Germany), project no FF20/2016 and have been performed in accordance with the Declaration of Helsinki. All methods were carried out in accordance with their relevant guidelines and regulations. Written informed consent was obtained from all study

participants. Furthermore, informed consent was obtained from the owner of the involved dog.

The animal study was carried out in compliance with the ARRIVE guidelines.

\section{Consent for publication}

Not applicable.

\section{Competing interests}

The authors have declared that they have no conflicts of interests.

\section{Author details}

'2nd Department of Internal Medicine, Municipal Hospital Darmstadt, Grafenstraße 9, 64283 Darmstadt, Germany. ${ }^{2}$ Toxicology and Chemotherapy Unit, German Cancer Research Center, Heidelberg, Germany. ${ }^{3}$ Institute for Medical Biostatistics, Epidemiology and Informatics, Johannes Gutenberg-University Mainz, Mainz, Germany. ${ }^{4}$ Pulmonologist's Office Darmstadt, Darmstadt, Germany. 
Received: 21 December 2020 Accepted: 26 July 2021

Published online: 13 August 2021

\section{References}

1. Koch-Institut R. Krebs in Deutschland 2015/2016. https://www.krebsdaten. de/Krebs/DE/Content/Publikationen/Krebs_in_Deutschland/kid_2019/krebs_ in_deutschland_2019.pdf?_blob=publicationFile.

2. Onkologie L. S3-Leitlinie Lungenkarzinom 2018. https://www.awmf.org/ uploads/tx_szleitlinien/020-007OL_I_S3_Lungenkarzinom_2018-03.pdf.

3. The National Lung Screening Trial Research Team. Reduced lung-cancer mortality with low-dose computed tomographic screening. N Engl J Med. 2011;365(5):395-409. https://doi.org/10.1056/NEJMoa1 102873.

4. De Konig $\mathrm{H}$, et al. Reduced lung-cancer mortality with volume $\mathrm{CT}$ screening in a randomized trial. N Engl J Med. 2020;382(6):503-13. https://doi.org/10.1 056/NEJMoa1911793.

5. Jenkins EK, DeChant MT, Perry EB. When the nose Doesn't know: canine olfactory function associated with health, management, and potential links to microbiota. Front Vet Sci. 2018;5:56. https://doi.org/10.3389/fvets.2018. 00056.

6. Robin S, Tacher S, Rimbault M, Vaysse A, Dréano S, André C, et al. Genetic diversity of canine olfactory receptors. BMC Genomics. 2009;10(1):21. https:// doi.org/10.1186/1471-2164-10-21.

7. McCulloch M, Jezierski T, Broffman M, Hubbard A, Turner K, Janecki T. Diagnostic accuracy of canine scent detection in early- and late-stage lung and breast cancers. Integr Cancer Ther. 2006;1:30-9.

8. Pirrone F, Albertini M. Olfactory detection of cancer by trained sniffer dogs: a systematic review of the literature. J Vet Behav. 2017;19:105-17. https:// doi.org/10.1016/j.jveb.2017.03.004

9. Ehmann R, Boedeker E, Friedrich U, Sagert J, Dippon J, Friedel G, et al. Canine scent detection in the diagnosis of lung cancer: revisiting a puzzling phenomenon. Eur Respir J. 2011;39:669-76.

10. Amundsen T, Sundstrom S, Buvik T, Gederaas OA, Haaverstad R. Can dogs smell lung cancer? First study using exhaled breath and urine screening in unselected patients with suspected lung cancer. Acta Oncol (Madr). 2014;53: 307-15.

11. Hackner K, Errhalt P, Mueller MR, Speiser M, Marzluf BA, Schulheim A, et al. Canine scent detection for the diagnosis of lung cancer in a screening-like situation. J Breath Res. 2016;10(4):046003. https://doi.org/10.1088/1752-71 55/10/4/046003

12. Jezierski T, Walczak M, Ligor T, Rudnicka J, Buszewski B. Study of the art: canine olfaction used for cancer detection on the basis of breath odour. Perspectives and limitations. J Breath Res. 2015;9(2).

13. Biehl W, Hattesohl A, Jörres RA, Duell T, Althöhn U, Rembert Koczulla A, et al. VOC pattern recognition of lung cancer: a comparative evaluation of different dog- and eNose-based strategies using different sampling materials. Acta Oncol. 2019;58(9):1216-24. https://doi.org/10.1080/02841 86X.2019.1634284.

14. Horvath G, Andersson H, Paulsson G. Characteristic odour in the blood reveals ovarian carcinoma. BMC Cancer. 2010;643.

15. Criee C, Baur X, Berdel D, Bosch D, Gappa M, Haidl P, et al. Spirometrie S2kLeitlinie der Deutschen Atemwegsliga, der Deutschen Gesellschaft für Pneumologie und Beatmungsmedizin und der Deutschen Gesellschaft für Arbeitsmedizin und Umweltmedizin zur Spirometrie. https:/www.awmf.org/ uploads/tx_szleitlinien/020-0171_S2k_Spirometrie-2015-05.pdf.

16. Liu J, Thomas PS. Relationship between exhaled breath condensate volume and measurements of lung volumes. Respiration. 2007;74(2):142-5. https:// doi.org/10.1159/000094238

17. Fischer-Tenhagen C, Johnen D, Nehls I, Becker R. A proof of concept: Are detection dogs a useful tool to verify potential biomarkers for lung cancer? Front Vet Sci. 2018;5(52).

18. Gordon RT, Schatz CB, Myers L, Kosty M, Gonczy C, Kroener J, et al. The use of canines in the detection of human cancers. J Altern Complement Med. 2008;14(1):61-7. https://doi.org/10.1089/acm.2006.6408.

19. Dammas S, Patz EF, Goodman PC. Identification of small lung nodules at autopsy: implications for lung cancer screening and overdiagnosis bias. Lung Cancer. 2001;33(1):11-6. https://doi.org/10.1016/S0169-5002(01)001908.

20. Walczak M, Jezierski T, Gorecka-Bruzda A, Sobczyńska M, Ensminger J. Impact of individual training parameters and manner of taking breath odor samples on the reliability of canines as cancer screeners. J Vet Behav. 2012; 7(5):283-94. https://doi.org/10.1016/j.jveb.2012.01.001.
21. Baldini C, Billeci L, Sansone F, Conte R, Domenici C. Tonacci a electronic nose as a novel method for diagnosing Cancer: a systematic review. Biosensors (Basel). 2020;10(8):84-93. https://doi.org/10.3390/bios10080084.

22. Li W, Dai W, Liu M, Long Y, Wang C, Xie S, et al. VOC biomarkers identification and predictive model construction for lung cancer based on exhaled breath analysis: research protocol for an exploratory study. BMJ Open. 2019;9(8):e028448. https://doi.org/10.1136/bmjopen-2018-028448.

23. Schallschmidt $K$, Becker R, Jung C, Bremser W, Walles T, Neudecker J, Leschber G, Frese S, Nehls I. Comparison of volatile organic compounds from lung cancer patients and healthy controls-challenges and limitations of an observational study. J Breath Res. 2016;10(4):046007. https://doi.org/1 0.1088/1752-7155/10/4/046007.

24. Rudnicka J, Walczak M, Kowalkowski T, Jezierski T, Buszewski B. Determination of volatile organic compounds as potential markers of lung cancer by gas chromatography-mass spectrometry versus trained dogs. Sensors Actuators B Chem. 2014;202:615-21. https://doi.org/10.1016/j.snb.2 014.06.006

25. Buszewski B, Ligor T, Jezierski T, Wenda-Piesik A, Walczak M, Rudnicka J. Identification of volatile lung cancer markers by gas chromatography-mass spectrometry: comparison with discrimination by canines. Anal Bioanal Chem. 2012;404(1):141-6. https://doi.org/10.1007/s00216-012-6102-8.

26. Deng C, Zhang X, Li N. Investigation of volatile biomarkers in lung cancer blood using solid-phase microextraction and capillary gas chromatographymass spectrometry. J Chromatogr B Anal Technol Biomed Life Sci. 2004; 808(2):269-77. https://doi.org/10.1016/j.jchromb.2004.05.015.

27. Sponring A, Filipiak W, Mikoviny T, Ager C, Schubert J, Miekisch W, et al. Release of volatile organic compounds from the lung cancer cell line $\mathrm{NCl}$ H2087 in vitro. Anticancer Res. 2009;29(1):419-26.

\section{Publisher's Note}

Springer Nature remains neutral with regard to jurisdictional claims in published maps and institutional affiliations.

\section{Ready to submit your research? Choose BMC and benefit from:}

- fast, convenient online submission

- thorough peer review by experienced researchers in your field

- rapid publication on acceptance

- support for research data, including large and complex data types

- gold Open Access which fosters wider collaboration and increased citations

- maximum visibility for your research: over $100 \mathrm{M}$ website views per year

At BMC, research is always in progress.

Learn more biomedcentral.com/submissions 